\title{
Stable Configurations of Carbon Clusters: Chains, Rings, and Fullerenes
}

\author{
H. Handschuh, G. Ganteför, B. Kessler, P. S. Bechthold, and W. Eberhardt \\ Forschungszentrum Jülich GmbH, Institut für Festkörperforschung, D-52425 Jülich, Germany
}

\begin{abstract}
A systematic study of the size dependence of the vibrational modes of carbon clusters with 570 atoms is presented. The vibrational frequencies are measured using photoelectron spectroscopy of mass-separated negatively charged clusters. The clusters are carefully annealed. Only the most stable isomers are present in the beam after the annealing. Observed vibrational modes can be assigned to four dominant isomeric structures: linear chains $\left(\mathrm{C}_{5}^{-}, \mathrm{C}_{7}^{-}\right.$, and $\left.\mathrm{C}_{9}^{-}\right)$, monocyclic rings $\left(\mathrm{C}_{10}^{-}, \mathrm{C}_{12}^{-}, \mathrm{C}_{14}^{-}\right.$, $\mathrm{C}_{16}^{-}$, and $\mathrm{C}_{18}^{-}$), bicyclic rings $\left(\mathrm{C}_{20}^{-}, \mathrm{C}_{24}^{-}\right.$, and $\mathrm{C}_{28}^{-}$), and fullerenes (even $n>\mathrm{C}_{30^{-}}{ }^{-}$).
\end{abstract}

PACS numbers: $36.40 .+\mathrm{d}, 33.60 . \mathrm{Cv}, 35.20 . \mathrm{Pa}$

The geometric structure of small carbon clusters is of basic interest since the discovery of the soccerball-like structure of $\mathrm{C}_{60}$. Many structures have been proposed for the clusters with less than 60 atoms: chains, monocyclic rings, polycyclic rings, fullerenes, and structures with lower symmetries. Related to this question is the nature of the growth process of fullerenes and whether ring structures may be stable precursors [1-3]. Many theoretical predictions deal with the structure of small carbon clusters, however, the experimental determination of the structure has turned out to be difficult except for $\mathrm{C}_{60}, \mathrm{C}_{70}$, some larger clusters like $\mathrm{C}_{76}[4]$, and some metallofullerenes $[5,6]$ for which macroscopic amounts can be generated and separated due to the solubility of the clusters. The measurement of the ion mobility, on the other hand, gives valuable data on the number and the dimensionality of isomers, but no detailed geometrical information on the ground state isomer $[1,2]$. Photoelectron spectroscopy of negatively charged carboncluster ions (anions) has been proven to provide important information on electronic properties $[7,8]$. Vibrational resolution, which gives information on geometric properties, has only been reached for the smallest carbon-cluster anions up to $\mathrm{C}_{11}^{-}$[9]. Our new experimental setup produces well-annealed carbon-cluster anions $\mathrm{C}_{n}^{-}$in the vibrational ground state up to $n=70$. Vibrational modes of the neutral clusters are excited by the electron-detachment process and can be resolved in the photoelectron spectra. Therefore this experimental method yields information similar to infrared and Raman spectroscopy without the need to prepare macroscopic amounts of mass selected cluster material. However, due to the different selection rules, different modes are observed. Here we apply this newly developed experimental capability to determine the structure of annealed carbon clusters.

The experimental setup $[10,11]$ consists of a modified laser-vaporization source, a time-of-flight (TOF) mass spectrometer, and excimer laser $(\mathrm{XeCl}, 4.025 \mathrm{eV})$ for electron detachment, and a time-of-flight electron spectrometer. The clusters are generated by laser vaporization of graphite. An annealing process right after the vaporization causes an enhancement of the relativistic intensities of the most stable isomers of the clusters. Thus, after the condensation of the $\mathrm{C}$ plasma into clusters, the clusters and $\mathrm{He}$ are heated by an electrical discharge and cooled down again in a long extender by subsequent supersonic expansion. Our view of this process is that the most stable isomers are formed by fragmentation and "annealing" induced by the additional energy of the discharge. Without the annealing step the photoelectron spectra do not display any electronic or vibronic structure. This is in agreement with the earlier findings of a mixture of various different isomers generated from laser vaporization [1,2]. The extender has been prolonged to a length of about $50 \mathrm{~cm}$ in order to achieve a proper cooling of the carbon clusters. Without proper cooling the spectra show an increased high intensity tail at low kinetic energies which can be attributed to thermionic emission [12,13]. The negatively charged clusters are mass separated by a Wiley-McLaren TOF mass spectrometer. A bunch of cluster anions of a certain mass is irradiated by a uv-laser pulse $(\mathrm{XeCl}$, $4.025 \mathrm{eV}$ ). The kinetic energy of the detached electrons is measured using a "magnetic-bottle" type time-of-flight electron spectrometer [14]. The energy resolution of the electron spectrometer varies with the kinetic energy of the electrons between 50 and $100 \mathrm{meV}$; it is about $30 \mathrm{meV}$ for most of the data presented here. The electron affinities of Ref. [9] for $n=5,7$, and 9 have been used to calibrate the energy scale. The absolute value of the binding energy scale is uncertain by $\pm 50 \mathrm{meV}$.

The mass spectrum of the anions displayed in Fig. 1 gives a first hint of the different structures of carbon clusters: The mass distribution shows low intensity for $n<10$, high intensity for $n=10$ to 18 , again very low intensity for $n=19-30$, and, finally, higher intensity for even numbers of $n$ with $n>30$. Since the clusters are well annealed, the relative intensity of a specific mass can be interpreted as a fingerprint of the stability of the cluster. Therefore the mass spectrum of the annealed clusters can be subdivided into four mass regimes:

$$
\begin{aligned}
& \text { (i) } n<10, \quad \text { (ii) } n=10-18, \quad \text { (iii) } n=20-28, \\
& \text { and (iv) } n>30 .
\end{aligned}
$$




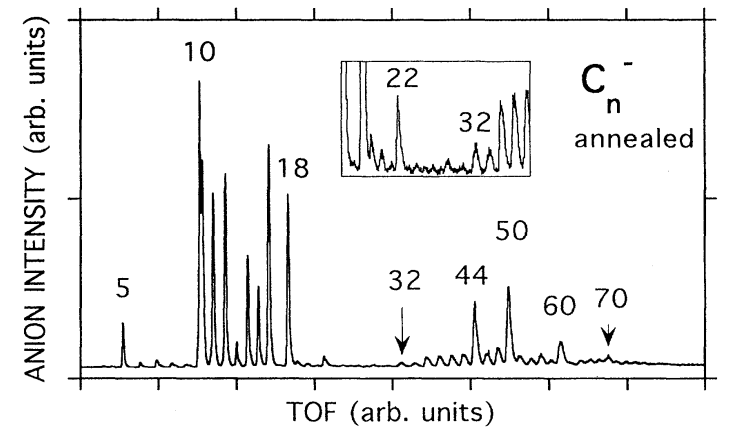

FIG. 1. Time-of-flight mass spectrum of annealed carbon cluster anions $\mathrm{C}_{n}^{-}$. Some cluster sizes $n$ are indicated by numbers. The inset shows the region for $n=16-40$, with an expanded $y$ scale $(\times 8)$

These four regimes, may correspond to certain preferred geometric structures. For example, it is proposed and supported by previous experimental and theoretical studies that a transition from a linear chain to a monocyclic ring structure takes place around $n=10[7,15-19]$.

Vibrationally resolved photoelectron spectra (PES) of these $\mathrm{C}_{n}^{-}$clusters provide direct insight into the geometric structure of each individual species. Figure 2 displays some spectra representative for the four regimes.
Indeed the spectra of clusters of the same regimes [columns (i), (ii), (iii), and (iv)] exhibit certain similarities indicating corresponding similarities of the geometric structures of the clusters. The spectra show several narrow (e.g., $\mathrm{C}_{9}^{-}$) or broad (e.g., $\mathrm{C}_{10}^{-}$) features which are assigned to electronic transitions from the ground state of the anion into the electronic states of the neutral cluster. The feature at lowest binding energy (BE), in most cases, corresponds to the transition into the neutral ground state. The fine structure of the features is interpreted in terms of the excitation of vibrations of the neutral cluster. A single vibrational progression consists of several equally spaced peaks (marked by bars). This spacing corresponds to the vibrational frequency (typically up to $2500 \mathrm{~cm}^{-1} \cong 0.3 \mathrm{eV}$ ) of a specific mode of the cluster that has been excited by the detachment process. The shape corresponds to the Franck-Condon profile of the transition between the anion and the neutral carbon cluster. An interpretation of these spectral features in terms of electronically excited states can be excluded, since all of the clusters discussed here are electronically closed shell species with highest-occupied-molecular-orbital-lowestunoccupied-molecular orbital gaps that are larger than 0.7 eV $[7,17,20-23]$.

Column (i) of Fig. 2 displays photoelectron spectra of $\mathrm{C}_{5}^{-}, \mathrm{C}_{7}^{-}$, and $\mathrm{C}_{9}^{-}$, which agree with earlier data [9].

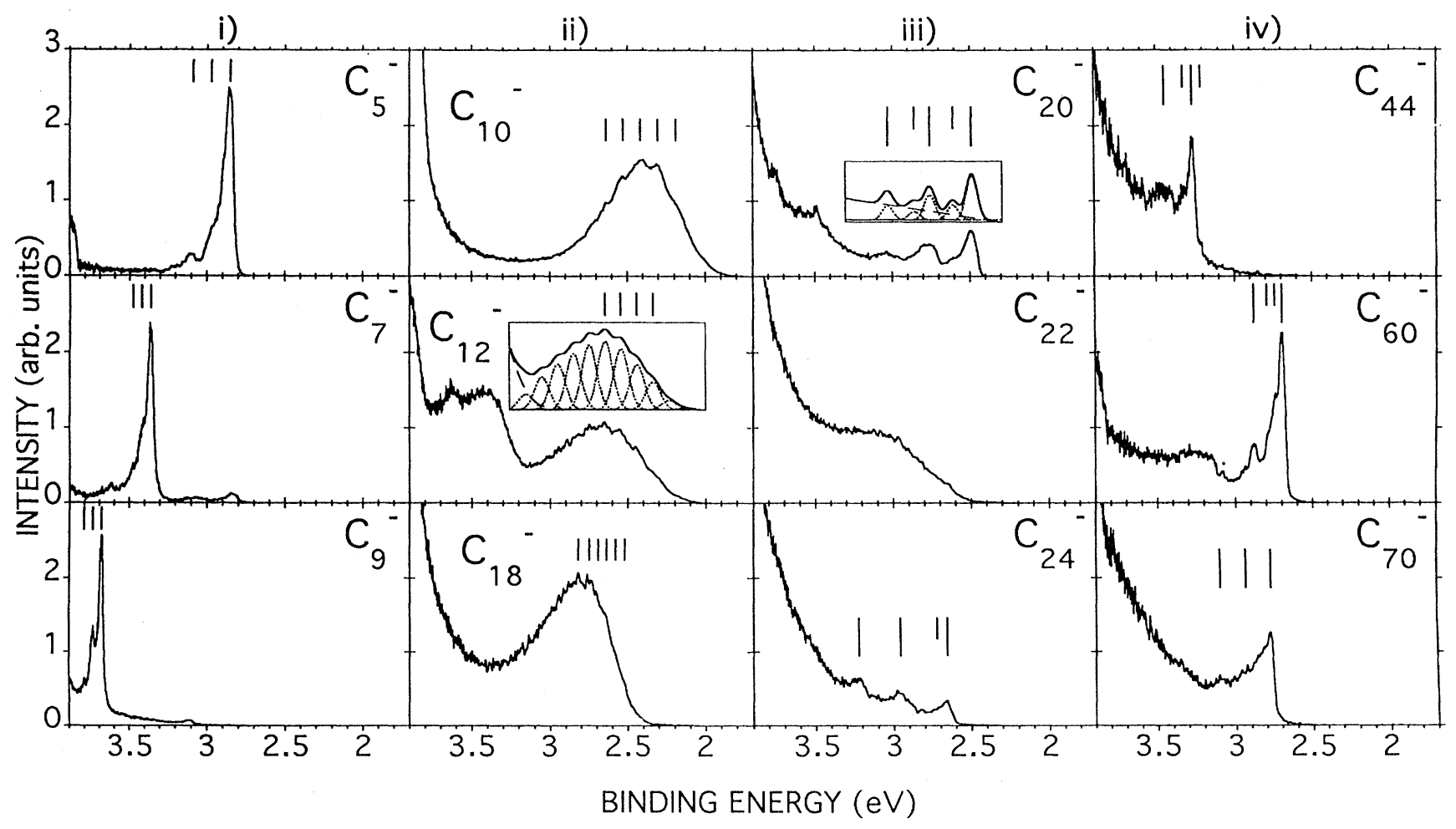

FIG. 2. Examples of photoelectron spectra of mass selected carbon cluster anions $\mathrm{C}_{n}^{-}$, as a function of the binding energy $(h \nu=4.025 \mathrm{eV}$; the insets show fits of the spectra with Gaussian curves at the positions of the bars plus a background): (i) $n=5$, 7, and 9; (ii) $n=10,12$, and 18; (iii) $n=20,22$, and 24; and (iv) $n=44,60$, and 70 . 
The strongest peak in the PES is assigned to the $0-0$ vibrational transition from the electronic ground state of the anion in a linear configuration to the neutral ground state $\left({ }^{2} \Sigma_{g}^{+} \rightarrow{ }^{1} \Sigma_{g}^{+}\right)$. The structures at the high binding energy side correspond to the excitation of symmetric stretch vibrations [9]. The frequencies corresponding to the vibrational progression are displayed in Fig. 3 as a function of cluster size $n$. The frequencies decrease with increasing cluster size due to the increasing mass [9].

Figure 2(ii) shows the photoelectron spectra of $\mathrm{C}_{10}^{-}$, $\mathrm{C}_{12}{ }^{-}$, and $\mathrm{C}_{18}{ }^{-}$. In contrast to the sharp peaks of Fig. 2(i), the main feature at lowest $\mathrm{BE}$ consists of a broad peak with vibrational fine structure. The inset displays a fit of the spectrum by Gaussian curves at the appropriate positions of the vibrational progression, illustrating the procedure used to determine the vibrational frequencies. These values are displayed in Fig. 3. The vibrations of $n=10,12,14,16$, and 18 do not simply prolong the series of the linear chains $(n=5,7$, and 9) but open a new branch of decreasing frequency with increasing cluster size.

Linear chains have unsaturated carbon bonds at the ends which can be avoided by building ring structures. With a growing number of atoms the strain that is caused by bending the cluster to a ring decreases. Starting with $\mathrm{C}_{10}{ }^{-}$, the strain becomes sufficiently small to make the ring an energetically favored structure [15]. The clusters with $n=10,12,14,16$, and 18 are quite likely to have the structure of planar monocyclic rings $[1,2,7,16,17,20]$. The frequencies are influenced by the bending strain on the bonds and by the mass of the cluster. Both these factors serve to explain the decrease of the frequency with increasing cluster size.

For $\mathrm{C}_{20}^{-}, \mathrm{C}_{24}^{-}$[Fig. 2(iii)], and $\mathrm{C}_{28}^{-}$(not shown) the structure of the photoelectron spectra is again completely different compared to the smaller clusters. Each spectrum

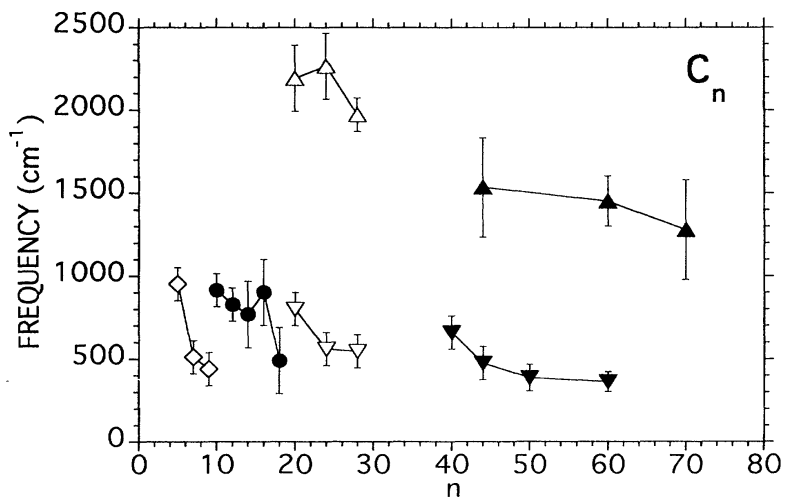

FIG. 3. Vibrational modes (taken from photoelectron spectra of $\mathrm{C}_{n}^{-}$) as a function of $n$. Open diamonds: linear carbon clusters, $n=5,7$, and 9; filled circles: monocyclic ring structures, $n=10,12,14,16$, and 18; open triangles: bicyclic ring structures, $n=20,24$, and 28 ; and filled triangles: fullerenes, $n=40,44,50,60$, and 70 . is dominated by three main peaks at low $\mathrm{BE}$ with an energy separation of almost $0.3 \mathrm{eV}$. The distances of the spectral features fit to two main vibrational progressions for each cluster size. The fit of the spectrum for $\mathrm{C}_{20}{ }^{-}$ (see inset) demonstrates that at least two frequencies contribute to the spectral features. The main frequencies are displayed in Fig. 3 and demonstrate the similarity of the three clusters. The low frequency mode does not simply continue the frequency branch of the monocyclic rings. This and the extraordinarily high frequency modes around $2000 \mathrm{~cm}^{-1}$ are striking differences in comparison with all other clusters discussed in this Letter.

Different predictions on the geometry of $\mathrm{C}_{20}$ have been made, ranging from linear structures, monocyclic rings, bicyclic structures, cuplike structures, to fullerenes $[3,24]$. The dramatic difference between $\mathrm{C}_{18}{ }^{-}$and $\mathrm{C}_{20}{ }^{-}$ can be explained by assuming that $C_{20}$ is the smallest cluster with a bicyclic structure. Mobility measurements on unannealed $\mathrm{C}$ clusters have revealed bicyclic structures in this size range, whereby the relative intensity of these isomers has been found to be very low $[19,24]$. Two structure models for bicyclic rings of $\mathrm{C}_{20}$ have been discussed [24]: (a) two rings of 10 carbon atoms joined by a biphenylene sort of structure (the carbon rings are expected to have alternating single and triple bonds "acetylenic," 900 and $2050 \mathrm{~cm}^{-1}$, respectively [25]), or (b) two rings of 11 carbon atoms joined by two cyclopropane structures. (The carbon rings are expected to have double bonds "cumulenic," $1650 \mathrm{~cm}^{-1}$ [25]). The high frequency of the triple bonds [model (a)] fits the data slightly better than model (b). Model (a) is restricted to $n=20,24$, and 28; 22 and 26 cannot be constructed as long as both rings are assumed to have the same size. The photoelectron spectra for $\mathrm{C}_{22}{ }^{-}$[see Fig. 2(iii)] and $\mathrm{C}_{26}{ }^{-}$(not shown) are strikingly different indeed, which also supports model (a).

Smaller clusters than $\mathrm{C}_{20}$ are not likely to follow the construction model for bicyclic rings because the strain makes smaller rings than $\mathrm{C}_{10}$ very unstable. A construction of larger bicyclic structures than $\mathrm{C}_{28}$ is not very likely because the fullerene structure becomes more stable with increasing cluster size. An interpretation that considers $\mathrm{C}_{20}{ }^{-}, \mathrm{C}_{24}{ }^{-}$, and $\mathrm{C}_{28}{ }^{-}$to be fullerenes is very unlikely since the symmetry of these small fullerene structures differs for $n=20\left(I_{h}\right.$, only pentagons, or $C_{2}$ due to Jahn-Teller distortion), $24\left(D_{6 d}\right)$, and $28\left(T_{d}\right.$, or $C_{1}$ due to Jahn-Teller distortion) $[22,26,27]$. However, the spectra clearly show similar structures. Calculations for $\mathrm{C}_{20}$ and $\mathrm{C}_{24}$ by density functional techniques including gradient corrections show that the fullerene is not energetically favored even when compared to monocyclic rings or bowl-shaped structures $[3,28]$.

We expect that a bicyclic ring may easily fragment into two monocyclic rings. This explains the low intensity of the clusters with $n=20,24$, and 28 in the mass spectrum (see Fig. 1). A dissociation study of carbon clusters has demonstrated that large monocyclic carbon cluster ring 
ions $\mathrm{C}_{n}^{+}(n>30)$ fragment by losing $\mathrm{C}_{10}, \mathrm{C}_{14}$, or $\mathrm{C}_{18}$ units [29]. Starting with model (a) for a bicyclic $\mathrm{C}_{20}$, for example, only two single bonds need to be broken to convert the cluster into two very stable monocyclic rings with $n=10$.

$\mathrm{C}_{n}{ }^{-}$clusters with even numbers of $n>30$ are expected to be fullerenes. This is confirmed by the mass spectrum (Fig. 1) due to the lack of odd numbered clusters and the high intensity of the magic numbers $\mathrm{C}_{44}{ }^{-}, \mathrm{C}_{50}{ }^{-}, \mathrm{C}_{60}{ }^{-}$, and $\mathrm{C}_{70}{ }^{-}$.

The photoelectron spectra of the most stable fullerenes $\mathrm{C}_{44}{ }^{-}, \mathrm{C}_{60}^{-}$, and $\mathrm{C}_{70}^{-}$[Fig. 2(iv)], on the other hand, give considerably more information. Low and high lying vibrational frequencies have been assigned tentatively and are plotted in Fig. 3. The higher frequencies around $1500 \mathrm{~cm}^{-1}$ are likely to belong to stretching vibrations within the surface of the fullerenes (tangential or angular motion) where the strong $\sigma$ bonds are located. The frequency does not change much for different fullerene sizes and clearly deviates from the high frequency branch of $n=20,24$, and 28. This is an additional indication to exclude an interpretation in terms of fullerenes for $\mathrm{C}_{20}{ }^{-}, \mathrm{C}_{24}{ }^{-}$, and $\mathrm{C}_{28}{ }^{-}$. The low frequency vibrations of the fullerenes around $500 \mathrm{~cm}^{-1}$ may correspond to breathingtype vibrations (radial motion) which are directed along the softer $\pi$ bonds. These frequencies are decreasing with increasing cluster size. This may be due to the fact that the $\pi$ bonds are less distorted for larger fullerenes. A tight binding calculation for $\mathrm{C}_{50}^{-}, \mathrm{C}_{60}{ }^{-}, \mathrm{C}_{70}{ }^{-}$, and $\mathrm{C}_{80}{ }^{-}$ predicts similar behavior [23]. A more careful analysis of vibrational properties has to take into account the symmetry of the clusters and goes beyond the scope of this Letter. Since the symmetry of $\mathrm{C}_{60}{ }^{-}$is well known, the spectrum of this cluster can be understood and will be given in more detail elsewhere [11]. The spectrum of $\mathrm{C}_{50}{ }^{-}$ is complicated (not shown), therefore a high frequency mode cannot be assigned unambiguously.

In conclusion, stable carbon cluster anions $\mathrm{C}_{n}^{-}$in the vibrational ground state are produced by an advanced laser vaporization technique. Spectroscopic evidence for different topological classes of structures is obtained by recording photoelectron spectra of mass selected carboncluster anions that exhibit a variety of vibrational frequencies. By comparison with the relative abundance in the mass spectra, an interpretation in terms of linear chains for $n=5,7$, and 9 monocyclic rings for even numbers of $n$ between 10 and 18, bicyclic rings for $n=20,24,28$, and fullerenes for even numbered clusters with $n>30$ is given.

[1] G.v. Helden, N.G. Gotts, and MT. Bowers, Nature (London) 363, 60 (1993).

[2] J. Hunter, J. Fye, and M. F. Jarrold, Science 260, 784 (1993).
[3] K. Raghavachari, D. L. Strout, G. K. Odom, G. E. Scuseria, J. A. Pople, B. G. Johnson, and P.M.W. Gill, Chem. Phys. Lett. 214, 357 (1993).

[4] R. Ettl, I. Chao, F. Diederich, and R. Whetten, Nature (London) 353, 149 (1991).

[5] K. Kikuchi, S. Suzuki, Y. Nakao, N. Nakahara, T. Wakabayashi, H. Shiromaru, K. Saito, I. Ikemoto, and Y. Achiba, Chem. Phys. Lett. 216, 67 (1993).

[6] R. Beyers, C-H. Kiang, R. D. Johnson, J. R. Salem, M.S. deVries, C.S. Yannoni, D. S. Bethune, H. C. Dorn, P. Burbank, K. Harich, and S. Stevenson, Nature (London) 370, 196 (1994).

[7] Shihe Yang, K. J. Taylor, M. J. Craycraft, J. Conceicao, C. L. Pettiette, O. Cheshnovsky, and R. E. Smalley, Chem. Phys. Lett. 144, 431 (1988).

[8] S. H. Yang, C. L. Pettiette, J. Conceicao, O. Cheshnovsky, and R. E. Smalley, Chem. Phys. Lett. 139, 233 (1987).

[9] D. W. Arnold, S.E. Bradforth, T. N. Kitsopoulos, and D. M. Neumark, J. Chem. Phys. 95, 8753 (1991).

[10] C-Y. Cha, G. Ganteför, and W. Eberhardt, Rev. Sci. Instrum. 63, 5661 (1992).

[11] O. Gunnarsson, H. Handschuh, P.S. Bechthold, B. Kessler, G. Ganteför, and W. Eberhardt (to be published).

[12] L-S. Wang, J. Conceicao, C. Jin, and R.E. Smalley, Chem. Phys. Lett. 182, 5 (1991).

[13] C. Yeretzian, K. Hansen, and R. L. Whetten, Science 260, 653 (1993).

[14] P. Kruit and F. H. Read, J. Phys. E 16, 313 (1983).

[15] G. v. Helden, P. R. Kemper, N. Gotts, and M. T. Bowers, Science 259, 1300 (1993).

[16] K. Raghavachari and J.S. Blinkley, J. Chem. Phys. 87, 2192 (1987).

[17] C. Liang and H.F. Schaefer, III, J. Chem. Phys. 93, 8844 (1990).

[18] V. Parasuk and J. Almlöf, Theor. Chim. Acta 83, 227 (1992).

[19] G. v. Helden, M. T. Hsu, N. Gotts, and M. T. Bowers, J. Phys. Chem. 97, 8182 (1993).

[20] K. S. Pitzer and E. Clementi, J. Am. Chem. Soc. 81, 4477 (1959)

[21] F. Jensen and H. Toftlund, Chem. Phys. Lett. 201, 89 (1993).

[22] B. L. Zhang, C.Z. Wang, K.M. Ho, C.H. Xu, and C.T. Chan, J. Chem. Phys. 97, 5007 (1992).

[23] S. J. Woo, E. Kim, and Y.H. Lee, Phys. Rev. B 47, 6721 (1993).

[24] G. v. Helden, M. T. Hsu, N. G. Gotts, P. R. Kemper, and M. T. Bowers, Chem. Phys. Lett. 204, 15 (1993).

[25] G. Herzberg, Molecular Spectra and Molecular Structure II (Van Nostrand Reinhold Company, New York, 1945), p. 195.

[26] H. W. Kroto and D. R. M. Walton, Chem. Phys. Lett. 214, 353 (1993).

[27] T. Guo, R. E. Smalley, and G. Scuseria, J. Chem. Phys. 99, 352 (1993).

[28] K. Raghavachari, B. Zhang, J. A. Pople, B. G. Johnson, and P. M. W. Gill, Chem. Phys. Lett. 220, 384 (1994).

[29] J. M. Hunter, J. L. Fye, and M. F. Jarrold, J. Chem. Phys. 99, 1785 (1993). 